\title{
Abundance segregation in Virgo spiral galaxies
}

\author{
O. L. Dors Jr. and M. V. F. Copetti
}

\begin{abstract}
Laboratório de Análise Numérica e Astrofísica, Departamento de Matemática, Universidade Federal de Santa Maria, 97119-900 Santa Maria, RS, Brazil

e-mail: oli@lana.ccne.ufsm.br
\end{abstract}

Received 25 November 2005 / Accepted 27 February 2006

\section{ABSTRACT}

\begin{abstract}
Aims. To derive $\mathrm{O} / \mathrm{H}, \mathrm{N} / \mathrm{O}$, and $\mathrm{S} / \mathrm{O}$ abundances of $\mathrm{H}$ II regions located in nine Virgo spiral galaxies, as well as to verify what is the cause of the abundance segregation found.

Methods. We employed photoionization models to reproduce observed emission-line intensities of $\mathrm{H}$ II regions located in the galaxies considered. The abundance gradients obtained were interpreted using a grid of chemical evolution models.

Results. Our models indicate that galaxies near to the core of the Virgo cluster are overabundant in O/H, N/O, and S/O by about $0.25 \mathrm{dex}$ in comparison to the ones at the periphery. With one exception, models with upper stellar mass limit of $M_{u}=30-40 M_{\odot}$ and age of the ionizing star cluster ranging from 1.5 to $2.5 \mathrm{Myr}$ were able to reproduce the observational data. Chemical models indicate the collapse time-scale for inner regions of galaxies near to the Virgo core is larger than the one in galaxies located at the intermediate and peripheral positions in the cluster, what can be due to the dense environment existing in Virgo Cluster core.
\end{abstract}

Key words. galaxies: abundances - galaxies: evolution - ISM: abundances - ISM: HII regions

\section{Introduction}

The chemical evolution of galaxies located in rich clusters is clearly influenced by their dense environment (see reviews by Haynes 1990 and Boselli \& Gavazzi 2006). Virgo cluster, due to its proximity, has served as the prototype system for studying intracluster medium and several theoretical and observational works have addressed the subject (e.g. Gunn \& Gott 1972; Chamaraux et al. 1980; Volmmer 2004).

Giovanelli \& Haynes (1985) found that galaxies in the Virgo Cluster are gas deficient in comparison with isolated galaxies. Besides, they found that the gas deficiency strongly correlates with the distance to the cluster center, in the sense that the galaxies in the inner parts are more gas depleted than the ones at the periphery. Warmels (1988) and Cayatte et al. (1994) found in some galaxies near to the Virgo Cluster center a gas deficiency more pronounced at large galactocentric radii. The main process invoked to explain the gas deficiency is the ram pressure stripping (Vollmer et al. 2001), which can lead to an increase of gas surface density in inner parts of the disk and to a removal of the gas in outer parts of that. Since the star formation is related to the gas content, gas deficient galaxies probably have suffered a different chemical evolution compared to their normal counterparts. In fact, van den Bergh (1976) found that cluster spiral galaxies have lower star formation rates than isolated spirals, result recently confirmed by Koopmann \& Kenney (2004) through $\mathrm{R}$ and $\mathrm{H} \alpha$ surface photometry in several Virgo and isolated galaxies. Moreover, spectrophotometric observations of H II regions in Virgo spiral galaxies (Skillman et al. 1996, and references therein) showed that galaxies located near the core are overabundant by 0.3 to $0.5 \mathrm{dex}$ in $\mathrm{O} / \mathrm{H}$ than the ones at the periphery. The Virgo abundance segregation has also been confirmed by Pilyugin et al. (2002) using the $P$-method (Pilyugin 2001).
Shields et al. (1991) and Skillman et al. (1996) argued that the Virgo overabundance can be partially due to the suppression of gas infall in galaxies located in Virgo core, making these galaxies to evolve more nearly as predicted by the closed box simple model for chemical evolution. However, Pilyugin et al. (2002) concluded that some isolated spirals have also evolved in a similar way, what makes the idea of infall curtailment at the cluster core somewhat doubtful.

The vast majority of the previous studies on chemical abundance gradients on Virgo spiral galaxies have only analyzed the behavior of the $\mathrm{O} / \mathrm{H}$ and $\mathrm{N} / \mathrm{O}$ ratios, and little attention has been given for $\mathrm{S} / \mathrm{O}$ gradients in these objects. The relative abundance between sulfur and oxygen can yield important informations about the functional form of the initial mass function and the mass range of the stellar cluster (Garnett 1989). In this paper we employed photoionization models to reproduce published emission line intensities of $\mathrm{H}$ II regions located in nine Virgo spiral galaxies in order to obtain their $\mathrm{O} / \mathrm{H}, \mathrm{N} / \mathrm{O}, \mathrm{S} / \mathrm{O}$ gradients, as well as the ionization parameter of the nebulae. Section 2 describes the modeling procedure, while the results are presented in Sect. 3. A comparison of our results with chemical evolution models and our final conclusions are given in Sects. 4 and 5, respectively.

\section{Photoionization models}

\subsection{Modeling procedure}

We employed the photoionization code Cloudy/95.03 (Ferland 2002) in order to reproduce the observational emission line intensities of seventy $\mathrm{H}$ II regions located in the following Virgo spiral galaxies: NGC 4501, NGC 4571, NGC 4689, NGC 4254, NGC 4321, NGC 4654, NGC 4303, NGC 4651, and NGC 4713. The observational data were taken from the papers by McCall et al. (1985), Shields et al. (1991), Henry et al. (1992, 1994), 
and Skillman et al. (1996). Only HII regions for which the lines [O II $] \lambda 3726+\lambda 3729$, [O III $] \lambda 5007,[\mathrm{~N} \mathrm{II}] \lambda 6584$, and [S II] $] 6716+\lambda 6731$ have been published were considered in our analysis. For this reason we selected only three objects in NGC 4689, NGC 4571, and NGC 4501. We adopted Skillman et al. (1996) classification, who divided the Virgo spirals into three groups in terms of the gas content: deficient (NGC 4501, NGC 4571, NGC 4689), intermediate (NGC 4254, NGC 4321, NGC 4654), and normal (NGC 4303, NGC 4651, NGC 4713). In each model a stellar cluster was assumed as the ionizing source. The stellar energy distributions (SEDs) obtained from the stellar population synthesis code STARBURST99 (Leitherer et al. 1999) were considered. We built a series of synthetic spectra assuming a cluster formed in an instantaneous burst, atmosphere models of Pauldrach et al. (2001), Salpeter initial mass function ( $\alpha=-2.35)$, upper stellar mass limits of $M_{\text {up }}=30,40,60$, $100 M_{\odot}$, metallicities $Z=2 \times Z_{\odot}, Z_{\odot}, Z_{\odot} / 2.5, Z_{\odot} / 5$, and age $(A)$ ranging from 0.01 to $10 \mathrm{Myr}$ with a step of $0.5 \mathrm{Myr}$. The solar composition refers to Grevesse \& Sauval (1998) and corresponds to $\log (\mathrm{O} / \mathrm{H})=-3.13, \log (\mathrm{N} / \mathrm{H})=-4.03$, and $\log (\mathrm{S} / \mathrm{H})=-4.79$. The metallicity of the nebula was matched with the closest available metallicity of the stellar cluster.

The nebula was considered static, plane parallel, with filling factor $\epsilon=0.01$ and electron density of $N_{\mathrm{e}}=200 \mathrm{~cm}^{-3}$. The model free parameters were basically (i) $\mathrm{O}, \mathrm{N}$, and $\mathrm{S}$ nebular abundances, (ii) $M_{\text {up }}$ and $A$, and (iii) the ionization parameter $U$, defined by $U=Q_{\mathrm{H}^{\circ}} / 4 \pi R_{\mathrm{s}}^{2} n c$; where $Q_{\mathrm{H}^{\circ}}$ is the number of hydrogen ionizing photons emitted per second by the ionizing cluster, $R_{\mathrm{S}}$ is the Strömgren radius, $n$ is the hydrogen density, and $c$ is the light speed. Models having different combinations of $Q_{\mathrm{H}^{\circ}}, N_{\mathrm{e}}$, and $\epsilon$, but with the same $U$ value are homologous and yield the same emission line intensities (Bresolin et al. 1999). The abundances of heavy metals were scaled with the oxygen abundance, with the exception of the $\mathrm{N}$ and $\mathrm{S}$ ones, which were free parameters. The presence of internal dust was considered and the grain abundances (van Hoof et al. 2001) were also linearly scaled with the oxygen abundance. To take depletion of refractory elements onto dust grains into account the abundances of the elements $\mathrm{Mg}$, $\mathrm{Al}, \mathrm{Ca}, \mathrm{Fe}, \mathrm{Ni}$, and $\mathrm{Na}$ were reduced by a factor of 10 , and $\mathrm{Si}$ by a factor of 2 (Garnett et al. 1995) relative to adopted abundances in each model.

We computed individual photoionization models for each H II region. The $M_{\text {up }}, A$, and $\log U$ parameters were chosen to be initially $40 M_{\odot}, 2 \mathrm{Myr}$, and -2.5 , respectively. As an initial guess we used the oxygen abundances derived from the index $R_{23}=([\mathrm{O}$ II $] \lambda 3727+[\mathrm{O}$ III $] \lambda 4949+\lambda 5007) / \mathrm{H} \beta($ Pagel et al. 1979) by Skillman et al. (1996), a solar S/O ratio, and nitrogen abundance taken from the relation $\log (\mathrm{N} / \mathrm{O})=\log (0.034+120 \mathrm{O} / \mathrm{H})$ of Vila-Costas \& Edmunds (1993). The input parameters were changed iteratively following a trial and error procedure until a satisfactory solution was found, in the sense that the predict emission line intensities reproduce the observational ones within the uncertainties.

\subsection{Uncertainties and uniqueness}

In order to evaluate the uncertainties and test the uniqueness of our models, we made several attempts to reproduce using different parameters the observational intensities of the emission line [O II] $\lambda 3726+\lambda 3729$, [O III] $\lambda 5007$, [N II] $] 6584$, and $[\mathrm{S} \mathrm{II}] \lambda 6716+\lambda 6731$ obtained by Peimbert (2003) for the well-resolved and nearby giant $\mathrm{HII}$ region 30 Doradus in the Large Magellanic Cloud. With the aim of varying all nebular

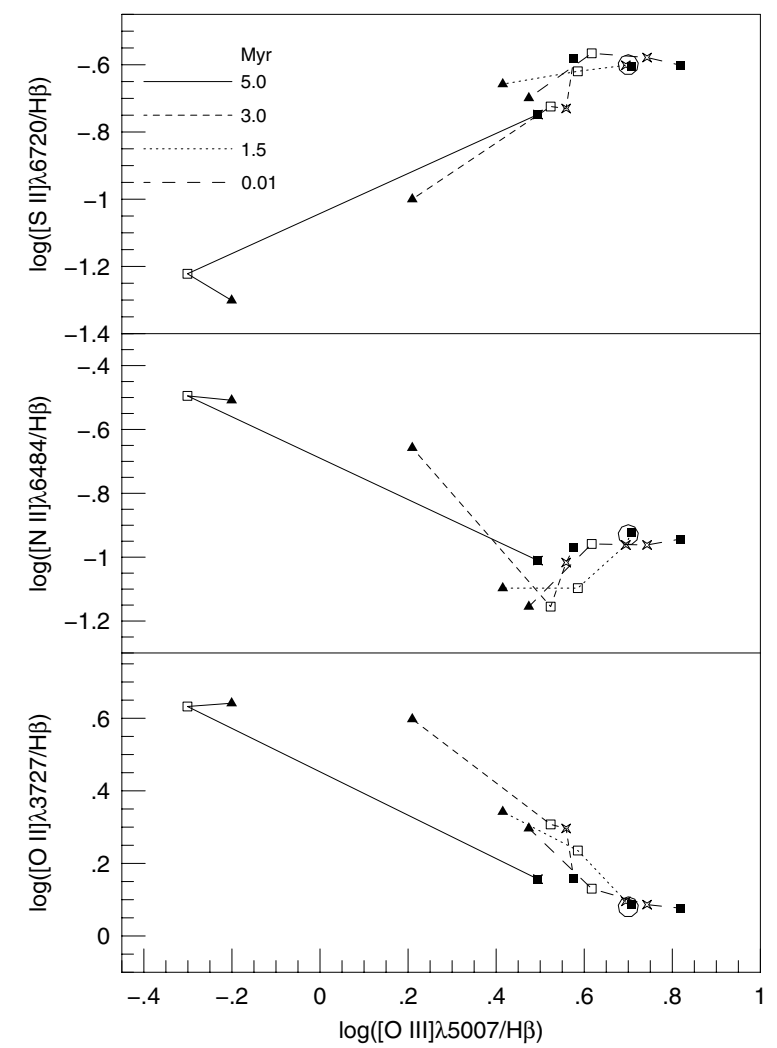

Fig. 1. The effects of different ages and stellar mass limits on the emission line intensities fitted for 30 Doradus. Lines connect models with different ages as indicated. The marks represent models with $M_{\text {up }}$ of

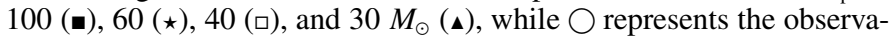
tional values for 30 Doradus obtained by Peimbert (2003).

parameters simultaneously, we used the routine of optimization Amoeba (Press et al. 1992) implemented in Cloudy.

We found an excellent match with the observational data with the model of $\log (\mathrm{O} / \mathrm{H})=-3.37, \log (\mathrm{N} / \mathrm{H})=-4.69$, $\log (\mathrm{S} / \mathrm{H})=-5.03, \log U=-2.24, M_{\text {up }}=100 M_{\odot}$, and age of 1.5 Myr. Our abundance results are about 0.2 dex larger than the ones derived by Peimbert (2003) via direct temperature determinations. The trend of overestimation of elemental abundances obtained from photoionization modelling of the strongest emission lines in comparison with direct determinations is not new and is discussed by Dors \& Copetti (2005, hereafter Paper I). The $M_{\text {up }}$ and $A$ parameters are compatible with those derived from spectroscopy of R136, the ionizing cluster of 30 Doradus, by Massey \& Hunter (1998).

To test the uncertainty of the nebular parameters, we tried to reproduce the 30 Doradus observational emission line intensities fixing the ionizing cluster parameters and varying the $\mathrm{O}, \mathrm{N}$, and $\mathrm{S}$ abundances and the ionization parameter. As result of this experiment we found that the elemental abundances and $U$ can be estimated with uncertainties of about 0.02 dex and 0.05 dex, respectively. A second test was made to evaluate the uncertainties in the determinations of $M_{\text {up }}$ and $A$. We considered all values of $M_{\text {up }}$ and $A$ presented in Sect. 2.1 and for each pair of these parameters, we tried to reproduce the observational data varying all nebular parameters (i.e. $U, \mathrm{O} / \mathrm{H}, \mathrm{N} / \mathrm{H}, \mathrm{S} / \mathrm{H}$ ) simultaneously. The abundance values varied freely on the range $2 \times Z_{\odot}-Z_{\odot} / 10$, without concern whether the final fitted values were consistent with the ones of our best fitting or with the values measured by Peimbert (2003). In Fig. 1 the results for this experiment are 
Table 1. Model parameters

\begin{tabular}{|c|c|c|c|c|c|c|}
\hline H II Region & $M_{\text {up }}\left(M_{\odot}\right)$ & $A$ (Myr) & $\log U$ & $12+\log (\mathrm{O} / \mathrm{H})$ & $\log (\mathrm{N} / \mathrm{O})$ & $\log (\mathrm{S} / \mathrm{O})$ \\
\hline $\begin{array}{l}+018,+012 \\
-043,-005 \\
-068,+093 \\
\end{array}$ & $\begin{array}{l}30 \\
30 \\
30 \\
\end{array}$ & $\begin{array}{l}2.0 \\
2.0 \\
2.0\end{array}$ & $\begin{array}{c}\text { NGC } 4501 \\
-2.50 \\
-2.70 \\
-2.40 \\
\end{array}$ & $\begin{array}{l}9.14 \\
9.07 \\
9.23 \\
\end{array}$ & $\begin{array}{l}-0.62 \\
-0.83 \\
-0.90 \\
\end{array}$ & $\begin{array}{l}-1.48 \\
-1.66 \\
-1.66 \\
\end{array}$ \\
\hline $\begin{array}{l}-017,-062 \\
+055,+018 \\
-010,+050 \\
\end{array}$ & $\begin{array}{l}30 \\
30 \\
30 \\
\end{array}$ & $\begin{array}{l}2.0 \\
2.0 \\
1.5 \\
\end{array}$ & $\begin{array}{c}\text { NGC } 4571 \\
-2.30 \\
-2.45 \\
-2.30 \\
\end{array}$ & $\begin{array}{l}9.01 \\
8.93 \\
9.00 \\
\end{array}$ & $\begin{array}{l}-0.68 \\
-0.85 \\
-0.90 \\
\end{array}$ & $\begin{array}{l}-1.41 \\
-1.42 \\
-1.41 \\
\end{array}$ \\
\hline $\begin{array}{l}+014,+007 \\
+001,-014 \\
-026,+001 \\
+048,-023 \\
-059,-013 \\
-077,-043 \\
+0131,+021\end{array}$ & $\begin{array}{l}30 \\
30 \\
40 \\
40 \\
40 \\
40 \\
40 \\
\end{array}$ & $\begin{array}{l}2.0 \\
2.0 \\
2.0 \\
2.0 \\
2.0 \\
2.0 \\
2.0\end{array}$ & $\begin{array}{l}\text { NGC } 4651 \\
-2.55 \\
-2.55 \\
-2.50 \\
-2.45 \\
-2.70 \\
-2.87 \\
-2.55 \\
\end{array}$ & $\begin{array}{l}9.11 \\
9.08 \\
9.02 \\
8.95 \\
8.77 \\
9.01 \\
8.86 \\
\end{array}$ & $\begin{array}{l}-0.82 \\
-0.85 \\
-1.05 \\
-1.10 \\
-0.94 \\
-1.29 \\
-1.12 \\
\end{array}$ & $\begin{array}{l}-1.66 \\
-1.75 \\
-1.88 \\
-1.95 \\
-1.58 \\
-2.05 \\
-1.70 \\
\end{array}$ \\
\hline $\begin{array}{l}-068,+033 \\
-034,-056 \\
-055,+051 \\
-042,+035 \\
+015,-029\end{array}$ & $\begin{array}{l}40 \\
40 \\
40 \\
40 \\
40\end{array}$ & $\begin{array}{l}2.0 \\
2.0 \\
2.0 \\
2.0 \\
2.0\end{array}$ & $\begin{array}{c}\text { NGC } 4654 \\
-2.62 \\
-2.45 \\
-2.50 \\
-2.70 \\
-2.54 \\
\end{array}$ & $\begin{array}{l}8.75 \\
8.56 \\
8.56 \\
8.95 \\
9.19 \\
\end{array}$ & $\begin{array}{l}-0.89 \\
-1.05 \\
-1.05 \\
-0.94 \\
-0.94 \\
\end{array}$ & $\begin{array}{l}-1.66 \\
-1.50 \\
-1.58 \\
-1.75 \\
-1.96 \\
\end{array}$ \\
\hline $\begin{array}{l}+016,-018 \\
-028,-019 \\
+014,+010\end{array}$ & $\begin{array}{l}30 \\
30 \\
30 \\
\end{array}$ & $\begin{array}{l}2.0 \\
2.0 \\
2.0 \\
\end{array}$ & $\begin{array}{c}\text { NGC } 4689 \\
-2.45 \\
-2.30 \\
-2.50 \\
\end{array}$ & $\begin{array}{l}9.07 \\
8.98 \\
8.96 \\
\end{array}$ & $\begin{array}{l}-0.70 \\
-0.68 \\
-0.89 \\
\end{array}$ & $\begin{array}{l}-1.41 \\
-1.42 \\
-1.41 \\
\end{array}$ \\
\hline $\begin{array}{l}-019,-022 \\
+012,-028 \\
+005,+014 \\
+042-002 \\
\end{array}$ & $\begin{array}{l}40 \\
40 \\
40 \\
40 \\
\end{array}$ & $\begin{array}{l}2.0 \\
2.0 \\
2.0 \\
2.0\end{array}$ & $\begin{array}{c}\text { NGC } 4713 \\
-2.25 \\
-2.45 \\
-2.45 \\
-2.65 \\
\end{array}$ & $\begin{array}{l}8.64 \\
8.71 \\
8.94 \\
8.47 \\
\end{array}$ & $\begin{array}{l}-1.05 \\
-1.48 \\
-1.20 \\
-1.34 \\
\end{array}$ & $\begin{array}{l}-1.66 \\
-1.66 \\
-1.66 \\
-1.58 \\
\end{array}$ \\
\hline $\begin{array}{l}-011,-018 \\
+031,-011 \\
-031,-022 \\
-007,-066 \\
+080,-016 \\
-039-076 \\
-039,-092 \\
+025,+100 \\
+106,+019 \\
-117,-002 \\
+077,+101 \\
+090,+102 \\
+013,+006 \\
-005,+042 \\
+055,-042 \\
-047,-075 \\
+102,+015\end{array}$ & $\begin{array}{l}30 \\
30 \\
30 \\
30 \\
40 \\
40 \\
40 \\
40 \\
40 \\
40 \\
40 \\
40 \\
30 \\
40 \\
40 \\
40 \\
40\end{array}$ & $\begin{array}{l}2.0 \\
2.0 \\
2.0 \\
2.0 \\
2.0 \\
2.0 \\
2.0 \\
2.0 \\
2.0 \\
2.0 \\
2.0 \\
2.0 \\
2.0 \\
2.0 \\
2.0 \\
2.0 \\
2.0\end{array}$ & $\begin{array}{l}\text { NGC } 4254 \\
-3.00 \\
-3.00 \\
-2.60 \\
-2.65 \\
-2.86 \\
-2.70 \\
-2.40 \\
-2.88 \\
-2.50 \\
-2.86 \\
-2.67 \\
-2.97 \\
-2.90 \\
-3.12 \\
-2.40 \\
-2.80 \\
-2.66\end{array}$ & $\begin{array}{l}9.33 \\
9.26 \\
9.31 \\
9.21 \\
9.14 \\
9.17 \\
8.79 \\
9.04 \\
8.93 \\
9.13 \\
8.98 \\
9.00 \\
9.32 \\
9.14 \\
8.99 \\
9.12 \\
9.05\end{array}$ & $\begin{array}{l}-1.20 \\
-1.12 \\
-1.02 \\
-1.12 \\
-1.05 \\
-1.02 \\
-0.94 \\
-1.12 \\
-0.92 \\
-1.05 \\
-1.26 \\
-1.15 \\
-0.90 \\
-0.94 \\
-0.74 \\
-0.96 \\
-1.05\end{array}$ & $\begin{array}{l}-1.96 \\
-2.05 \\
-1.88 \\
-1.88 \\
-1.96 \\
-1.88 \\
-1.48 \\
-1.93 \\
-1.63 \\
-1.88 \\
-1.86 \\
-1.93 \\
-2.00 \\
-1.75 \\
-1.59 \\
-1.84 \\
-1.84\end{array}$ \\
\hline
\end{tabular}

shown. Only models with $M_{\text {up }}=100-60 M_{\odot}$ and age of about 1.5 Myr match the observational data.

Changes in cluster synthetic spectra due to variations in the initial mass function parameters were analyzed by Mas-Hesse \& Kunth (1991), Leitherer (1994), and Leitherer \& Heckmam (1995). Such variations do not influence significantly abundance determinations via photoionization models, since the ionizing spectrum of an $\mathrm{H}$ II region depends mainly on the temperatures of the hottest stars in the ionizing cluster (Shields \& Tinsley 1976; Shields 1986) and even the spectrum of a single star of appropriate effective temperature when scaled in flux can mimic the ionizing radiation emitted by the cluster (Paper I).

\section{Results}

Table 1 presents the parameters for the final models. Column (1) lists the coordinate of each nebula, Cols. (2) and (3) list the adopted upper mass limit and the age of the stellar cluster, respectively. Column (4) lists the ionization parameter and Cols. (5)-(7) list the $\mathrm{O} / \mathrm{H}, \mathrm{N} / \mathrm{O}$, and $\mathrm{S} / \mathrm{O}$ abundance ratios. In Table 2 the predicted and observed line intensities are shown.

Our models reproduce the observational data assuming $M_{\text {up }}$ between 30 and $40 M_{\odot}$, with exception of $(-0.70,+140)$ in NGC 4303, for which we have to adopt $M_{\text {up }}=100 M_{\odot}$. This object has the smallest oxygen abundance of our sample and 
Table 1. continued.

\begin{tabular}{lcccccc}
\hline \hline H II Region & $M_{\text {up }}\left(M_{\odot}\right)$ & $A(\mathrm{Myr})$ & $\log U$ & $12+\log (\mathrm{O} / \mathrm{H})$ & $\log (\mathrm{N} / \mathrm{O})$ & $\log (\mathrm{S} / \mathrm{O})$ \\
\hline & & \multicolumn{5}{c}{ NGC 4303} \\
$+021,-007$ & 30 & 2.0 & -2.45 & 9.14 & -0.94 & -1.70 \\
$-001,+045$ & 30 & 2.0 & -2.60 & 9.15 & -0.85 & -1.88 \\
$-013,-044$ & 30 & 2.0 & -2.40 & 9.11 & -0.82 & -1.75 \\
$-014,+048$ & 30 & 2.0 & -2.30 & 9.11 & -0.78 & -1.75 \\
$+046,+006$ & 30 & 2.0 & -2.60 & 9.12 & -0.85 & -1.75 \\
$+032,-040$ & 40 & 2.0 & -2.58 & 8.91 & -0.70 & -1.75 \\
$+0.22,+067$ & 30 & 2.0 & -2.65 & 9.10 & -0.85 & -1.75 \\
$-049,-094$ & 40 & 2.0 & -2.35 & 8.71 & -0.99 & -1.66 \\
$-110,+075$ & 40 & 2.0 & -2.10 & 8.52 & -0.79 & -1.63 \\
$+010,-044$ & 30 & 2.0 & -2.60 & 9.21 & -1.15 & -1.88 \\
$-013,-044$ & 30 & 2.0 & -2.60 & 9.23 & -1.02 & -1.88 \\
$+045,-008$ & 30 & 2.0 & -2.60 & 9.14 & -1.21 & -2.00 \\
$-025,-042$ & 30 & 2.0 & -2.60 & 9.19 & -1.08 & -1.88 \\
$+031,-040$ & 40 & 2.0 & -2.80 & 9.11 & -1.15 & -1.96 \\
$+043,-026$ & 30 & 2.0 & -2.55 & 8.93 & -0.68 & -1.66 \\
$+016,-057$ & 30 & 2.0 & -2.60 & 9.19 & -1.08 & -1.88 \\
$+005,-073$ & 30 & 2.0 & -2.70 & 8.74 & -0.53 & -1.40 \\
$-008,-089$ & 30 & 2.0 & -2.25 & 8.68 & -1.05 & -1.58 \\
$-070,+140$ & 100 & 1.5 & -3.10 & 8.34 & -1.20 & -1.54 \\
\hline & & & NGC 4321 & & & \\
$+034,+145$ & 40 & 2.0 & -3.00 & 9.16 & -0.99 & -1.96 \\
$-001,-066$ & 30 & 2.0 & -2.50 & 8.86 & -0.82 & -1.38 \\
$+013,+102$ & 40 & 2.0 & -2.50 & 9.08 & -0.82 & -1.61 \\
$-032,+147$ & 40 & 2.0 & -2.72 & 8.89 & -0.94 & -1.70 \\
$-131,-027$ & 40 & 2.0 & -2.70 & 9.13 & -0.91 & -1.96 \\
$+029,+146$ & 40 & 2.0 & -2.90 & 9.17 & -1.05 & -2.0 \\
$+008,-004$ & 30 & 2.0 & -2.90 & 9.23 & -0.94 & -1.88 \\
$+032,-074$ & 30 & 2.0 & -2.90 & 9.24 & -0.90 & -1.88 \\
$-114,+010$ & 40 & 2.0 & -3.00 & 9.18 & -0.94 & -1.96 \\
\hline
\end{tabular}

the high value for the upper stellar mass limit is in consonance with the tendency for $M_{\text {up }}$ to increase with the decrease of $Z$ (Shields \& Tinsley 1976; Stasińska 1980; Vilchez \& Pagel 1988; Campbell 1988; Bresolin et al. 1999; Dors \& Copetti 2003, 2005). Ages of 1.5 or 2 Myr were assumed in the suitable models. Other papers considering stellar clusters as ionizing sources in order to reproduce strong forbidden lines of $\mathrm{H}$ II region have presented similar ages (e.g. Stasińska \& Izotov 2003; Bresolin et al. 1999; Copetti et al. 1985). Similar ages have also been found from optical photometric data of giant H II regions (e.g. Mayya \& Prabhu 1996). Selection effects may explain this limited range of ages. H II regions younger than about 1 Myr are difficult to detect in the optical, since they are generally embedded in dusty molecular clouds which cause considerable optical extinction. Nebulae older than about $5 \mathrm{Myr}$ are also difficult to observe because their original massive stars have cooled or are dead (Garcia-Vargas et al. 1996; Copetti et al. 1986). So, the age of 2 Myr was considered as a typical value for the nebulae analyzed and only when it was impossible to fit the data with this age a different value was adopted. However, we can not exclude the likely presence of older nebulae in our sample, and certainly a larger age spread than the one assumed in this paper is present. We found ionization parameters in the range $-3.1 \leq \log U \leq-2.1$, which agree with the ones found in Paper I for $\mathrm{H}$ II regions located in four isolated spiral galaxies.

In Fig. 2 we plot our estimates of N/O and S/O against the $\mathrm{O} / \mathrm{H}$ abundance for the sample of $\mathrm{H}$ II regions considered. Points corresponding to $\mathrm{H}$ II regions in gas deficient galaxies populate the upper right region of these diagrams, clearly indicating an abundance segregation. The gas deficient galaxies are in mean overabundant in $\mathrm{O} / \mathrm{H}, \mathrm{N} / \mathrm{O}$, and $\mathrm{S} / \mathrm{O}$ by about 0.25 dex in relation to the normal galaxies.
Figures 3-5 show our abundance results for $\mathrm{O} / \mathrm{H}, \mathrm{N} / \mathrm{O}$, and $\mathrm{S} / \mathrm{O}$ plotted as a function of galactocentric radius normalized to the effective radius taken from Skillman et al. (1996). Oxygen gradients found by Skillman et al. (1996) using the index $R_{23}$ and the oxygen calibrations of Edmunds \& Pagel (1984), O/H and N/O gradients using the $P$-method by Pilyugin et al. (2002), as well as gradients obtained using photoionization model sequences for NGC 4254 and NGC 4303 by Henry et al. (1992, 1994) are also plotted in Figs. 3-5.

Linear regression coefficients of our abundance gradients are shown in Table 3. Due to the small number of $\mathrm{H}$ II regions considered in NGC 4689, NGC 4571, and NGC 4501 the three gas deficient galaxies of our sample, no fitting was made for these objects. For them, our models predict oxygen values intermediate to the ones obtained via $R_{23}$ and $P$-method. For galaxies with normal and intermediate gas content, our oxygen gradients are consistent with the ones obtained via $R_{23}$ and via photoionization models by Henry et al. $(1992,1994)$. However, our O/H abundances are higher than the ones obtained via the $P$-method by Pilyugin et al. (2002). A similar discrepancy have been found in isolated galaxies (see Paper I). For NGC 4654, our O/H gradient is steeper than the gradients obtained by the other authors. We found the N/O gradients similar but slightly shallower than the ones obtained via $P$-method and Henry's photoionization models. The largest discrepancy between these different N/O estimates was for NGC 4654. In relation to the differential S/O abundance, for NGC 4654 we obtained a positive gradient, while for the other galaxies no clear gradient was found. Unfortunately only NGC 4254 and NGC 4303 have their S/O gradients estimated by Henry et al. (1992, 1994). As can be seen in Fig. 5, our S/O predictions for these two galaxies are consistent with the ones by Henry et al. (1992, 1994). 
Table 2. Predicted and observed emission line intensities in relation to $\mathrm{H} \beta=1.0$.

\begin{tabular}{|c|c|c|c|c|c|c|c|c|}
\hline \multirow[t]{2}{*}{ H II region } & \multicolumn{2}{|c|}{$[\mathrm{O}$ III $] \lambda 3726+\lambda 3729$} & \multicolumn{2}{|c|}{$\overline{[\mathrm{O}} \mathrm{III}] \lambda 5007$} & \multicolumn{2}{|c|}{$\overline{[\mathrm{N} \text { II }] \lambda 6584}$} & \multicolumn{2}{|c|}{ 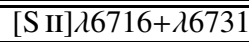 } \\
\hline & Obs. & Mod. & Obs. & Mod. & Obs. & Mod. & Obs. & Mod. \\
\hline \multicolumn{9}{|c|}{ NGC 4501 } \\
\hline$+018,+012$ & $0.22(0.07)$ & 0.30 & $\leq 0.09$ & 0.03 & $0.85(0.07)$ & 0.80 & $0.41(0.08)$ & 0.50 \\
\hline$-043,-005$ & $1.00(0.12)$ & 1.00 & $\leq 0.09$ & 0.09 & $0.94(0.07)$ & 1.00 & $0.63(0.07)$ & 0.71 \\
\hline$-068,+093$ & $0.63(0.08)$ & 0.69 & $\leq 0.06$ & 0.02 & $0.89(0.06)$ & 0.86 & $0.30(0.08)$ & 0.60 \\
\hline \multicolumn{9}{|c|}{ NGC 4571 } \\
\hline$-017,-062$ & $0.58(0.07)$ & 0.58 & $0.04(0.02)$ & 0.08 & $0.85(0.07)$ & 0.88 & $0.58(0.07)$ & 0.58 \\
\hline$+055,+018$ & $1.07(0.12)$ & 1.11 & $0.12(0.07)$ & 0.13 & $0.81(0.08)$ & 0.83 & $0.75(0.09)$ & 0.85 \\
\hline$-010,+050$ & $0.69(0.11)$ & 0.75 & $0.15(0.15)$ & 0.14 & $0.56(0.06)$ & 0.57 & $0.52(0.08)$ & 0.59 \\
\hline \multicolumn{9}{|c|}{ NGC 4651 } \\
\hline$+014,+007$ & $1.17(0.11)$ & 1.10 & $0.10(0.04)$ & 0.12 & $1.17(0.07)$ & 1.09 & $0.72(0.06)$ & 0.68 \\
\hline$+001,-014$ & $1.63(0.14)$ & 1.69 & $0.17(0.03)$ & 0.18 & $1.25(0.08)$ & 1.26 & $0.61(0.05)$ & 0.66 \\
\hline$-026,+001$ & $2.66(0.21)$ & 2.68 & $0.89(0.05)$ & 0.92 & $0.74(0.05)$ & 0.72 & $0.47(0.04)$ & 0.43 \\
\hline$+048,-023$ & $3.23(0.26)$ & 3.33 & $1.23(0.08)$ & 1.27 & $0.61(0.07)$ & 0.65 & $0.29(0.09)$ & 0.34 \\
\hline$-059,-013$ & $2.98(0.24)$ & 2.99 & $1.25(0.07)$ & 1.32 & $0.71(0.07)$ & 0.74 & $0.74(0.07)$ & 0.82 \\
\hline$-077,-043$ & $3.86(0.30)$ & 3.87 & $0.62(0.04)$ & 0.62 & $0.54(0.04)$ & 0.64 & $0.51(0.04)$ & 0.52 \\
\hline$+0131,+021$ & $2.85(0.23)$ & 2.90 & $0.99(0.06)$ & 0.96 & $0.59(0.08)$ & 0.58 & $0.63(0.09)$ & 0.60 \\
\hline \multicolumn{9}{|c|}{ NGC 4654} \\
\hline$-068,+033$ & $2.97(0.23)$ & 3.00 & $0.82(0.04)$ & 0.92 & $0.91(0.05)$ & 0.92 & $0.65(0.04)$ & 0.64 \\
\hline$-034,-056$ & $3.06(0.24)$ & 2.92 & $1.35(0.07)$ & 1.32 & $0.47(0.05)$ & 0.49 & $0.61(0.07)$ & 0.60 \\
\hline$-055,+051$ & $3.27(0.26)$ & 3.29 & $1.31(0.07)$ & 1.33 & $0.52(0.05)$ & 0.53 & $0.57(0.05)$ & 0.57 \\
\hline$-042,+035$ & $2.06(0.16)$ & 1.94 & $0.46(0.04)$ & 0.47 & $0.89(0.06)$ & 0.90 & $0.61(0.05)$ & 0.63 \\
\hline$+015,-029$ & $1.66(0.13)$ & 1.61 & $0.46(0.03)$ & 0.51 & $0.84(0.04)$ & 0.83 & $0.32(0.05)$ & 0.34 \\
\hline \multicolumn{9}{|c|}{ NGC 4689} \\
\hline$+016,-018$ & $0.48(0.10)$ & 0.42 & $\leq 0.07$ & 0.04 & $0.71(0.08)$ & 0.75 & $0.31(0.09)$ & 0.34 \\
\hline$-028,-019$ & $0.65(0.08)$ & 0.68 & $0.10(0.05)$ & 0.10 & $0.90(0.08)$ & 0.93 & $0.26(0.07)$ & 0.33 \\
\hline$+014,+010$ & $0.38(0.07)$ & 0.37 & $\leq 0.09$ & 0.03 & $0.63(0.06$ & 0.69 & $0.27(0.08)$ & 0.35 \\
\hline \multicolumn{9}{|c|}{ NGC 4713} \\
\hline$-019,-022$ & $2.86(0.22)$ & 2.89 & $1.77(0.09)$ & 1.83 & $0.44(0.02)$ & 0.45 & $0.39(0.02)$ & 0.35 \\
\hline$+012,-028$ & $3.36(0.26)$ & 3.31 & $1.34(0.07)$ & 1.36 & $0.22(0.03)$ & 0.22 & $0.59(0.05)$ & 0.53 \\
\hline$+005,+014$ & $2.38(0.26)$ & 2.29 & $0.82(0.04)$ & 0.85 & $0.47(0.05)$ & 0.42 & $0.57(0.06)$ & 0.57 \\
\hline$+042-002$ & $3.80(0.30)$ & 3.97 & $2.05(0.10)$ & 2.13 & $0.27(0.05)$ & 0.24 & $0.51(0.08)$ & 0.63 \\
\hline \multicolumn{9}{|c|}{ NGC 4254 } \\
\hline$-011,-018$ & $0.32(0.08)$ & 0.37 & $0.08(0.03)$ & 0.04 & $0.30(0.02)$ & 0.28 & $0.28(0.02)$ & 0.26 \\
\hline$+031,-011$ & $0.88(0.14)$ & 0.83 & $0.04(0.04)$ & 0.04 & $0.57(0.04)$ & 0.58 & $0.40(0.03)$ & 0.39 \\
\hline$-031,-022$ & $0.63(0.07)$ & 0.62 & $0.07(0.02)$ & 0.09 & $0.54(0.02)$ & 0.53 & $0.35(0.02)$ & 0.34 \\
\hline$-007,-066$ & $1.42(0.13)$ & 1.48 & $0.17(0.02)$ & 0.14 & $0.73(0.03)$ & 0.72 & $0.54(0.02)$ & 0.57 \\
\hline$+080,-016$ & $1.96(0.31)$ & 1.99 & $0.37(0.04)$ & 0.35 & $0.88(0.09)$ & 0.85 & $0.49(0.05)$ & 0.52 \\
\hline$-039-076$ & $1.45(0.32)$ & 1.52 & $0.36(0.03)$ & 0.37 & $0.71(0.03)$ & 0.73 & $0.48(0.02)$ & 0.47 \\
\hline$-039,-092$ & $1.89(0.23)$ & 1.90 & $0.87(0.05)$ & 0.85 & $0.60(0.03)$ & 0.59 & $0.64(0.04)$ & 0.63 \\
\hline$+025,+100$ & $3.09(0.43)$ & 2.99 & $0.48(0.06)$ & 0.48 & $0.80(0.08)$ & 0.85 & $0.65(0.07)$ & 0.63 \\
\hline$+106,+019$ & $2.06(0.32)$ & 2.03 & $0.74(0.07)$ & 0.73 & $0.71(0.07)$ & 0.77 & $0.54(0.05)$ & 0.59 \\
\hline$-117,-002$ & $1.79(0.28)$ & 1.80 & $0.32(0.03)$ & 0.32 & $0.78(0.08)$ & 0.80 & $0.67(0.07)$ & 0.60 \\
\hline$+077,+101$ & $3.26(0.14)$ & 3.20 & $0.80(0.03)$ & 0.80 & $0.56(0.02)$ & 0.53 & $0.60(0.02)$ & 0.58 \\
\hline$+090,+102$ & $3.29(0.50)$ & 3.35 & $0.42(0.07)$ & 0.42 & $0.83(0.10)$ & 0.84 & $0.75(0.09)$ & 0.71 \\
\hline$+013,+006$ & $0.41(0.08)$ & 0.45 & $0.04(0.01)$ & 0.05 & $0.67(0.08)$ & 0.61 & $0.25(0.02)$ & 0.23 \\
\hline$-005,+042$ & $1.12(0.12)$ & 1.09 & $0.07(0.02)$ & 0.10 & $0.86(0.11)$ & 0.89 & $0.78(0.06)$ & 0.80 \\
\hline$+055,-042$ & $1.33(0.22)$ & 1.29 & $0.48(0.04)$ & 0.52 & $0.94(0.10)$ & 0.89 & $0.55(0.04)$ & 0.48 \\
\hline$-047,-075$ & $1.59(0.36)$ & 1.62 & $0.31(0.03)$ & 0.33 & $0.84(0.08)$ & 0.89 & $0.53(0.05)$ & 0.57 \\
\hline$+102,+015$ & $2.44(0.30)$ & 2.39 & $0.63(0.03)$ & 0.62 & $0.73(0.07)$ & 0.77 & $0.55(0.04)$ & 0.55 \\
\hline
\end{tabular}

\section{Comparison with chemical evolution models}

In order to interpret our abundance results presented in Sect. 3 and verify the cause of the abundance segregation found, we have employed the grid of multiphase chemical evolution models for galaxies (taken from website http://wwwae.ciemat.es/ mercedes) by Molla \& Diaz (2005) to reproduce our abundance gradients. These models have the advantage of using few input parameters: (i) the galaxy total mass $M_{\text {gal }}$; (ii) and the efficiencies to form molecular clouds $\left(\epsilon_{\mu}\right)$ and stars $\left(\epsilon_{\mathrm{H}}\right)$, which are connected by $\ln \epsilon_{\mu} / \ln \epsilon_{\mathrm{H}}=0.4$. This grid consists a set of models representing galaxies with 44 different total masses and considering 10 different efficiency values.
These models give, among other outputs, abundances of 14 elements along the galactic disk considering a step from 1 to $4 \mathrm{kpc}$, which depends of $M_{\mathrm{gal}}$, being larger for the most massive galaxies, and smaller for the less ones.

The following methodology to select what evolution models from the Molla \& Diaz's grid represent the galaxies analyzed in this paper was used. Initially, we selected models with masses compatible with that of the galaxy considered (see Table 4). For that, we used Table 1 of Molla \& Diaz (2005), which relates $M_{\text {gal }}$ with the maximum rotation velocity $V_{\max }$, which was taken from Skillman et al. (1996). From Table 5 of Molla \& Diaz's grid, which relates elemental abundances (in mass) with the collapse time-scale to form the galaxy disk $\tau_{\text {coll }}$ and efficiencies, we 
Table 2. continued.

\begin{tabular}{|c|c|c|c|c|c|c|c|c|}
\hline \multirow[t]{2}{*}{ H II region } & \multicolumn{2}{|c|}{$[\mathrm{O}$ II $] \lambda 3726+\lambda 3729$} & \multicolumn{2}{|c|}{ [O III] $] \lambda 5007$} & \multicolumn{2}{|c|}{$[\mathrm{N}$ II $] \lambda 6584$} & \multicolumn{2}{|c|}{$[\mathrm{S}$ II $] \lambda 6716+\lambda 6731$} \\
\hline & Obs. & Mod. & Obs. & Mod. & Obs. & Mod. & Obs. & Mod. \\
\hline \multicolumn{9}{|c|}{ NGC 4303} \\
\hline$+021,-007$ & $1.22(0.37)$ & 1.28 & $0.13(0.07)$ & 0.16 & $0.82(0.15)$ & 0.89 & $0.51(0.10)$ & 0.60 \\
\hline$-001,+045$ & $0.93(0.15)$ & 1.03 & $0.07(0.03)$ & 0.11 & $0.95(0.10)$ & 0.97 & $0.37(0.05)$ & 0.42 \\
\hline$-013,-044$ & $1.01(0.16)$ & 1.01 & $0.14(0.02)$ & 0.14 & $1.01(0.10)$ & 0.96 & $0.48(0.04)$ & 0.44 \\
\hline$-014,+048$ & $1.02(0.16)$ & 1.02 & $0.17(0.03)$ & 0.15 & $1.04(0.10)$ & 1.01 & $0.44(0.05)$ & 0.39 \\
\hline$+046,+006$ & $0.82(0.13)$ & 0.86 & $0.09(0.05)$ & 0.09 & $0.91(0.09)$ & 0.86 & $0.50(0.07)$ & 0.51 \\
\hline$+032,-040$ & $1.78(0.29)$ & 1.81 & $0.50(0.05)$ & 0.55 & $1.20(0.12)$ & 1.19 & $0.37(0.04)$ & 0.46 \\
\hline$+022,+067$ & $0.99(0.16)$ & 1.00 & $0.08(0.04)$ & 0.08 & $0.91(0.09)$ & 0.93 & $0.51(0.05)$ & 0.57 \\
\hline$-049,-094$ & $2.82(0.45)$ & 2.85 & $1.46(0.15)$ & 1.48 & $0.62(0.06)$ & 0.58 & $0.44(0.05)$ & 0.43 \\
\hline$-110,+075$ & $2.92(0.36)$ & 2.82 & $1.96(0.11)$ & 1.90 & $0.40(0.05)$ & 0.38 & $0.61(0.10)$ & 0.53 \\
\hline$+010,-044$ & $1.56(0.12)$ & 1.64 & $0.12(0.05)$ & 0.17 & $0.70(0.09)$ & 0.69 & $0.52(0.05)$ & 0.57 \\
\hline$-013,-044$ & $1.29(0.12)$ & 1.31 & $0.15(0.02)$ & 0.14 & $0.79(0.04)$ & 0.83 & $0.48(0.05)$ & 0.52 \\
\hline$+045,-008$ & $2.84(0.90)$ & 2.83 & $0.23(0.12)$ & 0.25 & $0.77(0.23)$ & 0.79 & $0.53(0.10)$ & 0.54 \\
\hline$-025,-042$ & $1.65(0.17)$ & 1.74 & $2(0.03)$ & 0.18 & $0.79(0.06)$ & 0.83 & $0.54(0.05)$ & 0.58 \\
\hline$+031,-040$ & $2.51(0.56)$ & 2.42 & $0.45(0.07)$ & 0.48 & $0.70(0.14)$ & 0.71 & $0.51(0.10)$ & 0.53 \\
\hline$+043,-026$ & $1.84(0.60)$ & 1.82 & $0.25(0.19)$ & 0.19 & $1.80(0.82)$ & 1.71 & $0.76(0.50)$ & 0.72 \\
\hline$+016,-057$ & $1.74(0.61)$ & 1.74 & $0.19(0.07)$ & 0.18 & $0.83(0.16)$ & 0.83 & $0.55(0.15)$ & 0.58 \\
\hline$+005,-073$ & $3.16(1.50)$ & 3.10 & $0.25(0.20)$ & 0.26 & $0.80(0.44)$ & 0.77 & $0.75(0.20)$ & 1.00 \\
\hline$-008,-089$ & $3.02(0.61)$ & 3.13 & $0.57(0.11)$ & 0.53 & $0.67(0.07)$ & 0.73 & $0.65(0.10)$ & 0.58 \\
\hline$-070,+140$ & $4.51(0.70)$ & 4.24 & $2.64(0.26)$ & 2.34 & $0.41(0.10)$ & 0.46 & $1.04(0.11)$ & 1.02 \\
\hline \multicolumn{9}{|c|}{ NGC 4321} \\
\hline$+034,+145$ & $1.59(0.20)$ & 1.61 & $0.20(0.03)$ & 0.21 & $0.96(0.13)$ & 0.95 & $0.55(0.05)$ & 0.55 \\
\hline$-001,-066$ & $1.06(0.21)$ & 1.14 & $0.16(0.06)$ & 0.12 & $0.85(0.08)$ & 0.88 & $0.96(0.11)$ & 0.96 \\
\hline$+013,+102$ & $1.03(0.17)$ & 0.97 & $0.27(0.06)$ & 0.31 & $0.74(0.07)$ & 0.75 & $0.51(0.05)$ & 0.51 \\
\hline$-032,+147$ & $2.60(0.42)$ & 2.71 & $0.57(0.12)$ & 0.64 & $0.90(0.09)$ & 0.95 & $0.78(0.06)$ & 0.73 \\
\hline$-131,-027$ & $1.87(0.30)$ & 1.88 & $0.48(0.05)$ & 0.47 & $1.02(0.10)$ & 1.03 & $0.45(0.05)$ & 0.42 \\
\hline$+029,+146$ & $1.87(0.30)$ & 1.84 & $0.32(0.03)$ & 0.30 & $0.89(0.09)$ & 0.85 & $0.47(0.03)$ & 0.48 \\
\hline$+008,-004$ & $0.82(0.17)$ & 0.80 & $\leq 0.04$ & 0.04 & $0.80(0.11)$ & 0.82 & $0.48(0.05)$ & 0.54 \\
\hline$+032,-074$ & $0.71(0.08)$ & 0.69 & $0.05(0.02)$ & 0.04 & $0.85(0.09)$ & 0.84 & $0.52(0.05)$ & 0.49 \\
\hline$-114,+010$ & $1.42(0.16)$ & 1.38 & $0.15(0.3)$ & 0.18 & $1.00(0.10)$ & 0.99 & $0.55(0.04)$ & 0.52 \\
\hline
\end{tabular}

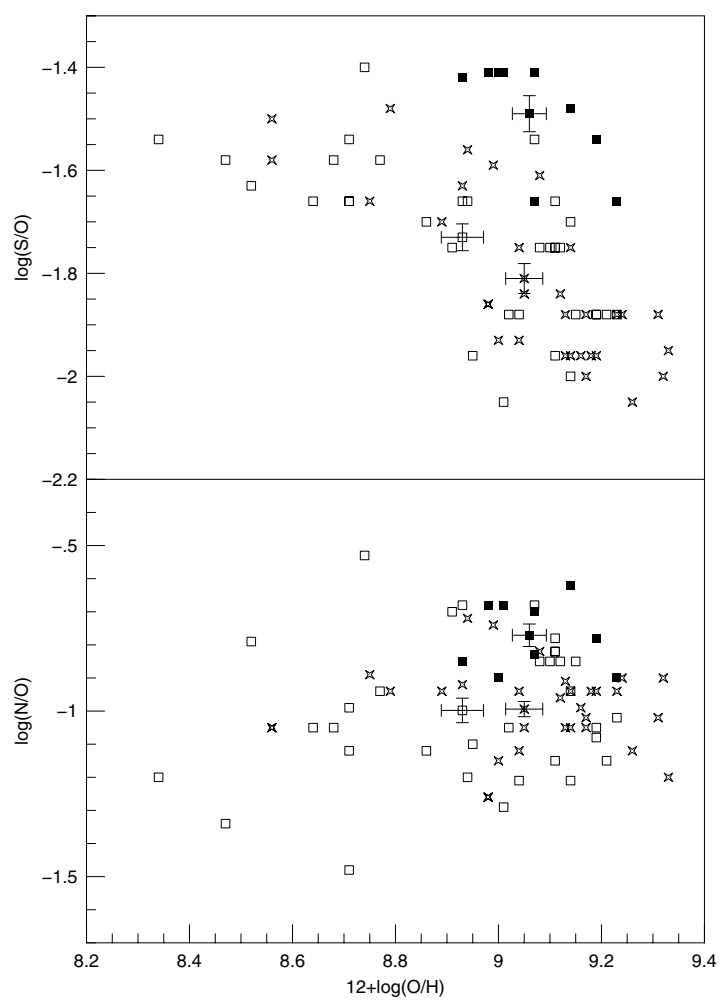

Fig. 2. $\mathrm{N} / \mathrm{O}$ and $\mathrm{S} / \mathrm{O}$ ratios vs. $\mathrm{O} / \mathrm{H}$ for the Virgo spiral galaxies. The filled squares, open squares, and the star symbols represent our abundance results for nebulae located in galaxies with abundances of $\mathrm{H} \mathrm{I}$ deficient, normal, and intermediate, respectively. The error bars represent the mean standard deviation for our abundance results. selected the models with a given $\tau_{\text {coll }}$ and efficiencies that result $\mathrm{N} / \mathrm{O}$ values along the galactic radii which better reproduce the N/O gradient shown in Table 3. We noted that chemical models with $\epsilon_{\mu} \geq 0.30$ and $\epsilon_{\mathrm{H}} \geq 0.05$ yield very similar abundances. These are typical values for bright galaxies (Molla \& Dias 2005), as those considered in this paper. So the $\epsilon_{\mu}=0.65$ and $\epsilon_{\mathrm{H}}=$ 0.34 mean efficiency values were adopted. The model selected gives us the $\mathrm{O} / \mathrm{H}, \mathrm{N} / \mathrm{O}, \mathrm{S} / \mathrm{O}$ ratios and $\tau_{\text {coll }}$ across the disk for each galaxy. Constant efficiency values were considered along the galaxy disk. A distance of $16.8 \mathrm{Mpc}$ for the Virgo cluster was assumed.

The reliability of direct oxygen determinations as well as of other elements (e.g. N, S, Ar) in metal-rich nebulae is an open question (Pilyugin et al. 2006; Stasińska 2005; Pilyugin 2003). Moreover, Molla \& Diaz (2005) pointed out that their models show sometimes bad fit to observations in the central parts of galaxy disks, once in those regions oxygen abundances higher than the oxygen saturation level of the models $(12+\log (\mathrm{O} / \mathrm{H}) \approx$ 9.0-9.1) are measured, such as in the case of central parts of Virgo galaxies. However, even though abundance determination of individual elements are uncertain, the ratios between abundances of heavy elements are more accurate. In fact, N/O abundances obtained via photoionization models agree with the ones estimated via direct electron temperature determinations and via $P$-method (Pilyugin et al. 2003) for a large range of abundance (Paper I). Because of that, we do not use $\mathrm{O} / \mathrm{H}$ as observational constraint but N/O instead.

Figures 3-5 show that the chemical models reproduce fairly well our $\mathrm{N} / \mathrm{O}$ and $\mathrm{S} / \mathrm{O}$ ratios, but not the $\mathrm{O} / \mathrm{H}$ ratio. The $\mathrm{S} / \mathrm{O}$ positive gradient derived by us for NGC 4654 is not reproduced by the chemical models. Direct abundance estimates of the 
Table 3. Linear regression coefficients $\left(X=m R / R_{E}+b\right)$ for abundance gradients.

\begin{tabular}{|c|c|c|c|c|c|c|}
\hline$\overline{\text { Galaxy }}$ & \multicolumn{2}{|c|}{$\overline{\mathrm{O} / \mathrm{H}}$} & \multicolumn{2}{|c|}{$\mathrm{N} / \mathrm{O}$} & \multicolumn{2}{|c|}{$\mathrm{S} / \mathrm{O}$} \\
\hline & $m$ & $\bar{b}$ & $m$ & $b$ & $m$ & $b$ \\
\hline NGC 4254 & $-0.13(0.03)$ & $9.29(0.06)$ & $-0.04(0.05)$ & $-0.94(0.07)$ & $0.04(0.05)$ & $-1.88(0.08)$ \\
\hline NGC 4321 & $-0.08(0.06)$ & $9.25(0.09)$ & $-0.03(0.04)$ & $-0.88(0.05)$ & $-0.03(0.09)$ & $-1.81(0.12)$ \\
\hline NGC 4654 & $-0.66(0.18)$ & $9.51(0.19)$ & $-0.10(0.09)$ & $-0.86(0.09)$ & $0.43(0.08)$ & $-2.14(0.08)$ \\
\hline NGC 4303 & $-0.36(0.04)$ & $9.36(0.04)$ & $-0.09(0.07)$ & $-0.83(0.07)$ & $0.12(0.05)$ & $-1.86(0.05)$ \\
\hline NGC 4651 & $-0.07(0.03)$ & $9.08(0.05)$ & $-0.11(0.05)$ & $-0.86(0.07)$ & $-0.02(0.07)$ & $-1.76(0.10)$ \\
\hline NGC 4713 & $-0.42(0.18)$ & $9.28(0.25)$ & $-0.16(0.31)$ & $-1.03(0.44)$ & $0.03(0.06)$ & $-1.69(0.09)$ \\
\hline
\end{tabular}

Table 4. Collapse time-scale ( $\left.\tau_{\text {coll }}\right)$ in Gyr for $\epsilon_{\nu}=0.65$ and $\epsilon_{\mathrm{H}}=0.34$.

\begin{tabular}{lcccccccccc}
\hline \hline \multirow{2}{*}{ Galaxy } & Mass $\left(10^{9} M_{\odot}\right)$ & \multicolumn{1}{c}{ Radius $(\mathrm{kpc})$} \\
& & 2 & 3 & 4 & 6 & 8 & 9 & 12 & 15 & 18 \\
\hline NGC 4501 & 5274 & - & - & 1.8 & - & 2.9 & - & - & - & - \\
NGC 4571 & 981 & 1.4 & - & - & - & - & - & - & - & - \\
NGC 4689 & 1377 & 2.5 & - & - & - & - & - & - & - & - \\
NGC 4254 & 5274 & - & - & 0.5 & - & 0.8 & - & 2.3 & - & - \\
NGC 4321 & 1791 & - & 0.7 & - & 1.4 & - & 4.1 & 6.1 & 8.9 & 13.2 \\
NGC 4654 & 1791 & - & - & 1.4 & 2.7 & 4.3 & - & - & - & - \\
NGC 4303 & 2220 & - & 1.0 & - & 1.8 & - & 4.2 & 6.6 & 10.5 & 12.8 \\
NGC 4651 & 3347 & - & 0.5 & - & 0.6 & - & 0.9 & - & - & - \\
NGC 4713 & 608 & 0.5 & - & - & - & - & - & - & - & - \\
\hline
\end{tabular}

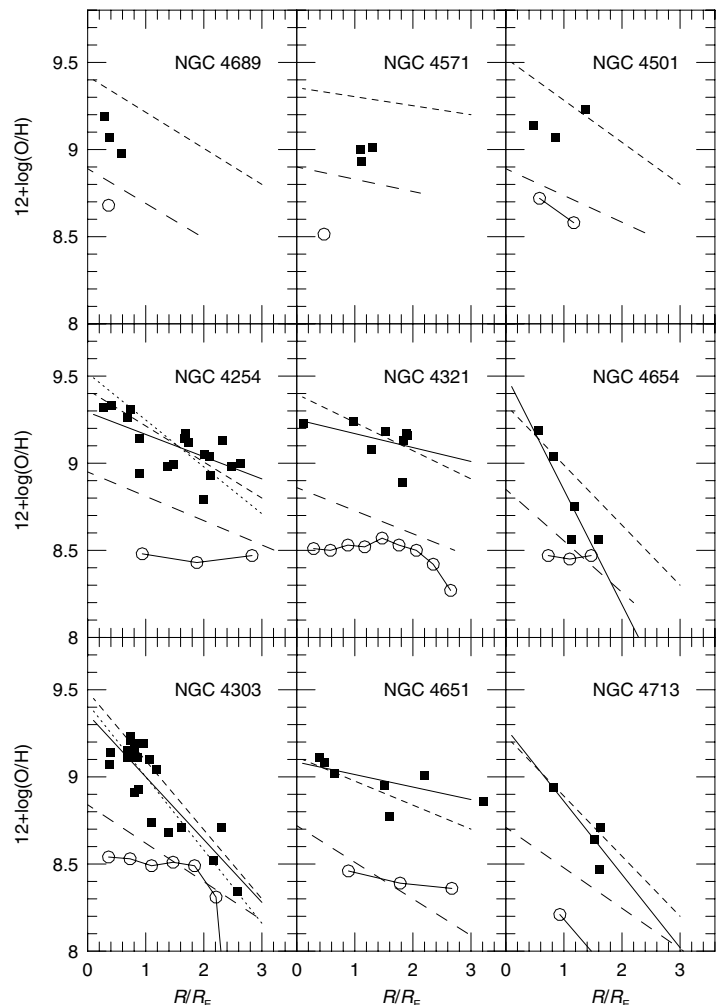

Fig. 3. Oxygen abundances vs. the galactocentric radius normalized to the effective radius for nine Virgo spiral galaxies. The solid lines are linear regressions of our model results represented by squares. Short dashed lines represent gradients obtained by Skillman et al. (1996) using the index $R_{23}$. Long dashed lines represents gradients via $P$-method by Pilyugin et al. (2002), while dotted lines represent gradients from photoionization models by Henry et al. $(1992,1994)$. The connected open circles represent the chemical models selected (see Sect. 4).

S/O ratio (e.g. Smith 1975; Torres-Peimbert et al. 1989; Garnett 1989; Garnett et al. 1997; Kennicutt et al. 2003; Izotov et al. 2004; Bresolin et al. 2004) showed no dependence on the

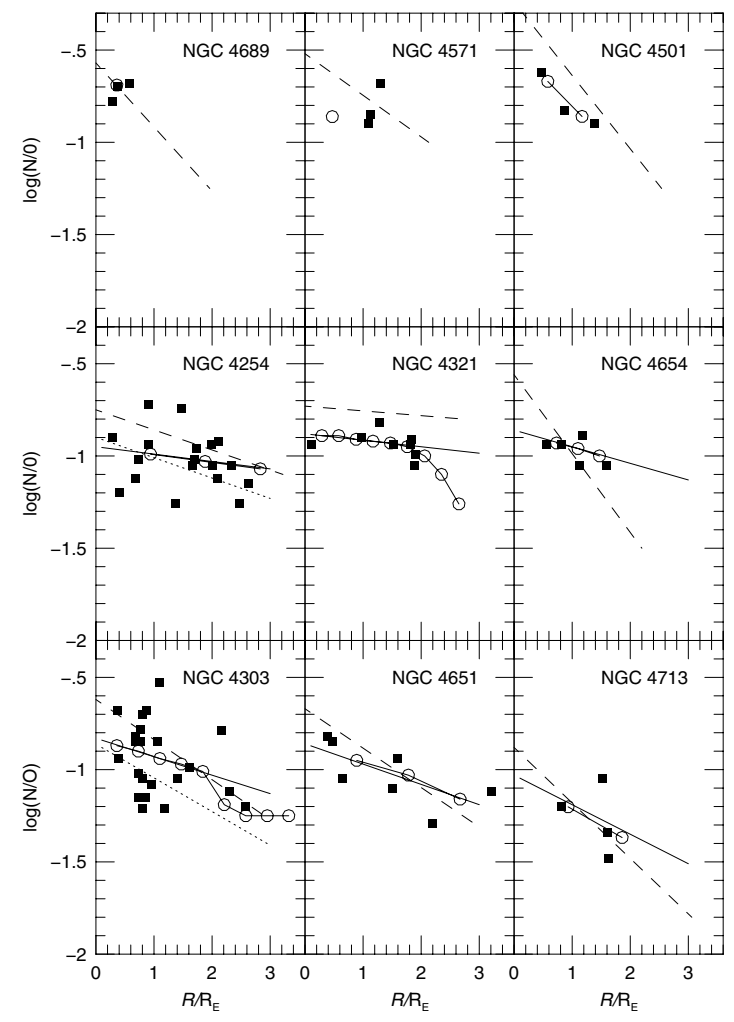

Fig. 4. Same as Fig. 3 but for $\log (\mathrm{N} / \mathrm{O})$.

galactocentric distance. So, we suspect that the S/O gradient found in NGC 4654 can be an artefact of the modeling procedure.

Table 4 presents the values of $\tau_{\text {coll }}$ along the radius for the galaxies considered. The galaxies located at the cluster center tend to have higher collapse time-scale than the other for a given radius. If we consider inner regions as being the ones with $R<4 \mathrm{kpc}$, galaxies with deficient, intermediate, and normal gas content have mean $\tau_{\text {coll }}$ of about $1.9,0.86$, and $0.66 \mathrm{Gyr}$, 


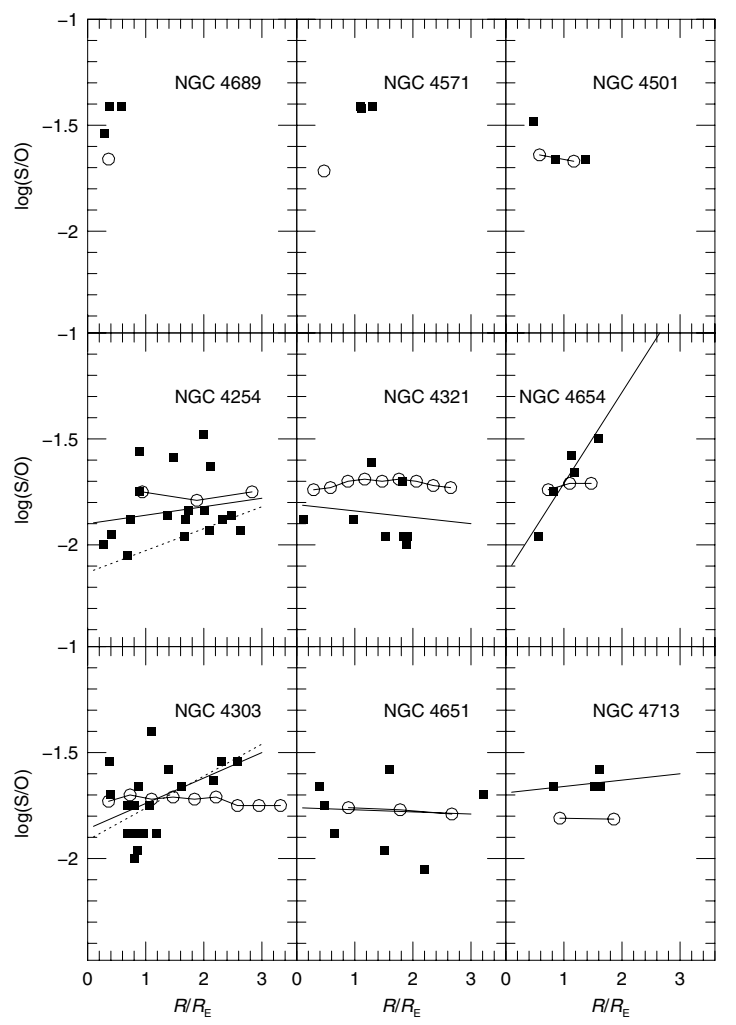

Fig. 5. Same as Fig. 3 but for $\log (\mathrm{S} / \mathrm{O})$.

respectively. Low-mass galaxies tend to form their disk in a timescale larger than the ones with high-mass (Molla \& Dias 2005). However, as there is no correlation between galaxy mass and the gas content in the sample the galaxies analyzed, the result above is an indication that the dense environment existing in the Virgo nucleus can inhibit the gas infall from halo, resulting in high metal abundances, as pointed out by Shields et al. (1991) and Skillman et al. (1996).

Pilyugin et al. (2002) computed detailed chemical evolution models for the Virgo galaxies NGC 4303, NGC 4321, and NGC 4501. They found that the inner regions of NGC 4501 and NGC 4321 would have very strong infall rate during the first two Gyr, with the infall finishing at 5 Gyr. NGC 4303 would present a slower infall lasting until now. For all galaxies analyzed by Pilyugin et al. (2002) we have derived lower $\tau_{\text {coll }}$ values than the ones derived by them. Our analysis do not extent to the field spiral, so we can not confirm the result found by Pilyugin et al. (2002) that some isolated spiral evolved in the same way as the Virgo spirals do.

\section{Conclusion}

We have reproduced emission-line intensities of $\mathrm{H}$ II regions located in nine Virgo spiral galaxies using photoionization models. Our results indicate that galaxies near to the core of the Virgo cluster are overabundant in $\mathrm{O} / \mathrm{H}, \mathrm{N} / \mathrm{O}$, and $\mathrm{S} / \mathrm{O}$ by about 0.25 dex in relation to the galaxies located at the periphery or at intermediate radius in the cluster. The upper stellar mass limits and the ages of the ionizing clusters of the nebulae are of the order of 30-40 $M_{\odot}$ and 1.5 to $2.5 \mathrm{Myr}$, respectively. Employing a grid of chemical evolution models for galaxies we concluded that the abundance segregation found in the considered Virgo galaxies can be attributed to the fact that the collapse timescale in gas deficient galaxies is larger than the ones in galaxies with normal and intermediate gas content. This result supports the idea proposed by Shields et al. (1991) and Skillman et al. (1996) that galaxies located in a dense environment tend to evolve nearly as predicted by the closed box model for chemical evolution.

Acknowledgements. We thank Dr. Gary Ferland for making the Cloudy programme available as well as the anonymous referee for many useful comments. We gratefully acknowledge Dra. Mercedes Molla for helping with the grid of chemical models and to the LSC (Laboratório de Sistemas de Computação, UFSM), where the calculations were performed. This work was supported by the Brazilian institutions CAPES and FAPERGS.

\section{References}

Boselli, A., \& Gavazzi, G. 2006 [arXiv: astro-ph/0601108] Bresolin, F., Kennicutt, R. C., \& Garnett, D. R. 1999, ApJ, 510, 104 Bresolin, F., Garnett, D. R., \& Kennicutt, R. C 2004, ApJ, 615, 228 Campbell, A. 1988, ApJ, 335, 644

Chamaraux, P., Balkowski, C., \& Gérard, E. 1980, A\&A, 83, 38 Cayate, V., Kotanyi, C., Balkowski, C., \& van Gorkom, J. H. 1994, AJ, 100, 604 Copetti, M. V. F., Pastoriza, M. G., \& Dottori, H. A. 1985, A\&A, 152, 427 Copetti, M. V. F., Pastoriza, M. G., \& Dottori, H. A. 1986, A\&A, 156, 111 Dors, O. L., \& Copetti, M. V. F. 2003, A\&A, 404, 969 Dors, O. L., \& Copetti, M. V. F. 2005, A\&A, 437, 837 Edmunds, M. G., \& Pagel, B. E. J. 1984, MNRAS, 211, 507

Evans, I. N. 1986, ApJ, 309, 544

Ferland, G. J. 2002, Hazy, a brief introduction to Cloudy 96.03, Univ. Kentucky, Dept. Phys., Astron. internal report

García-Vargas, M. L., Bressan, A., \& Díaz, A. I. 1996, A\&AS, 112, 13

Garnett, D. R. 1989, 345, 282

Garnett, D. R., Dufour, R. J., Peimbert, M., et al. 1995, ApJ, 44 9, 77

Garnett, D. R., Shields, G. A., Skillman, E. D., Sagan, S. P., \& Dufour, R. J. 1997, ApJ, 489, 63

Giovanelli, R., \& Haynes, M. 1985, ApJ, 292, 404

Grevesse, N., \& Sauval, A. 1998, Space Sci. Rev., 85, 161

Gunn, J. E., \& Gott, J. R. 1972, ApJ, 176, 1

Haynes, M. P. 1990, in Cluster of Galaxies, ed. W. R. Oegede, M. J. Fitchett, \& L. Danly (Cambridge: Cambridge Univ. Press), 177

Henry, R. C. B., Pagel, B. E. J., Lasseter, D. F., \& Chincarini, G. L. 1992, MNRAS, 258, 321

Henry, R. C. B., Pagel, B. E. J., \& Chincarini, G. L. 1994, MNRAS, 266, 421

Izotov, Y. I., Stasińska, G., Guseva, N. G., \& Thuan, T. X. 2004, A\&A, 415, 87 Kennicutt, R. C., Bresolin, F, \& Garnett, D. R. 2003, ApJ, 591, 801

Koopmann, R. A., \& Kenney, J. D. P. 2004, ApJ, 613, 851

Leitherer, C. 1994, Rev. Mod. Astron., 7, presented at the Annual meeting of the Astronomische Gesellshaft, Bochum, October 1993

Leitherer, C., \& Heckmam, T. M. 1995, ApJS, 96, 9

Leitherer, C., Schaerer, D., Goldader, J. D., et al. 1999, ApJS, 123, 3

Lequeux, J. 1983, A\&A, 125, 394

McCall, M. L., Rybski, P. M., \& Shields, G. A. 1985, ApJS, 57, 1

Massey, P., \& Hunter, D. A. 1998, ApJ, 493, 180

Mas-Hesse, J. M., \& Kunth, D. 1991, A\&AS, 88, 399

Mayya, Y. D., \& Prabhu, T. P. 1996, AJ, 111, 125

Molla, M., \& Diaz, A. I. 2005, MNRAS, 358, 521

Pagel, B. E. J., Edmunds, M. G., Blackwell, D. E., Chun, M. S., \& Smith, G. 1979, MNRAS 189,95

Pauldrach, A. W. A., Hoffmann, T. L., \& Lennon, M. 2001, A\&A, 375, 161

Peimbert, A. 2003, ApJ, 584, 735

Pilyugin, L. S. 2001, A\&A, 369, 594

Pilyugin, L. S. 2003, A\&A, 399, 1003

Pilyugin, L. S., Mollá, M., Ferrini, F., \& Vilchez, J. M. 2002, A\&A, 383, 14

Pilyugin, L. S., Thuan, T. X., \& Vilchez, J. M. 2003, A\&A, 397, 487

Pilyugin, L. S., Thuan, T. X., \& Vílchez, J. M. 2006 [arXiv: astro-ph/0601122]

Press, W. H., Teukolsky, S. A., Vetterling, W. T., \& Flannery, B. P. 1992, Numerical Recipes (Cambridge: Cambridge University Press)

Shields, G. A. 1986, PASP, 98, 1072

Shields, G. A., \& Tinsley, B. M. 1976, ApJ, 203, 66

Shields, G. A., Skillman, E. D., \& Kennicutt, R. C. 1991, ApJ, 371, 82

Skillman, E. D., Kennicutt, R. C., Shields, G. A., \& Zaritsky, D. 1996, ApJ, 462, 147

Smith, H. E. 1975, ApJ, 199, 591

Stasińska, G. 1980, A\&A, 84, 320

Stasińska, G. 2005, A\&A, 434, 507

Stasińska, G., \& Izotov, I. 2003, A\&A, 397, 71

Torres-Peimbert, S., Peimbert, M., \& Fierro, J. 1989, ApJ, 345, 186

van den Bergh, S. 1976, ApJ, 206, 883

van Hoof, P. A. M., Weingartner, J. C., Martin, P. G., Volk, K., \& Ferland, G. J. 2001, in Challenges of Photoionized Plasmas, ed. G. Ferland, \& D. Savin (San Francisco: ASP), ASP Conf Ser., 247,363

Vila-Costas, M. B., \& Edmunds, M. G. 1993, MNRAS, 265, 199

Vilchez, J. M., \& Pagel, B. E. J. 1988, MNRAS, 231, 257

Vollmer, B. 2003, A\&A, 398, 525

Vollmer, B., Cayatte, V., Balkowski, C., \& Duschl, W. J. 2001, ApJ, 561, 708

Warmels, R. H. 1988, ApJSS, 72, 427 